\title{
White-opaque switching in Candida albicans
}

\author{
Matthew B. Lohse ${ }^{1}$ and Alexander D. Johnson ${ }^{1,2,{ }^{*}}$ \\ ${ }^{1}$ Department of Biochemistry and Biophysics, University of California San Francisco, San Francisco, \\ California, United States of America \\ 2Department of Microbiology and Immunology, University of California San Francisco, San \\ Francisco, California, United States of America
}

\section{Summary}

The human commensal yeast Candida albicans undergoes an epigenetic switch between two distinct types of cells, referred to as white and opaque. These two cell types differ in many respects, including their cell and colony morphologies, their metabolic states, their mating behaviors, their preferred niches in the host, and their interactions with the host immune system. Each of the two cell types is heritable for many generations and switching between them appears stochastic; however, environmental cues can significantly alter the frequency of switching. We review recent work on white-opaque switching, including the establishment of the transcriptional circuit underlying this switch, the identification of environmental signals that affect switching rates, newly discovered differences between the two types of cells, and the involvement of white-opaque switching in biofilm formation. We also review recent speculation on the evolution and adaptive value of white-opaque switching.

\section{Introduction}

Candida albicans, the most common human fungal pathogen, resides asymptomatically in the gastrointestinal tract of most healthy humans. However, when the host immune system is compromised due to age, disease, or medical treatment, C. albicans can cause a range of medical problems; including disseminated blood-born infections with mortality rates exceeding 40\% [1]. C. albicans differs from the great majority of fungal species (and even from some very closely related species) by possessing the ability to switch between two distinctive types of cells, white and opaque. Each cell type is heritable for many generations and switching occurs without a change in the DNA sequence of the genome (hence our use of the term 'epigenetic's) [2••-6]. Under standard laboratory conditions switching is rare (approximately every $10^{4}$ cell divisions) although, as will be discussed, environmental factors can strongly influence the switching rate [7]. White and opaque cell types have distinct properties which result largely from the differential regulation of approximately 400 genes, about seven percent of the genome [8,9]. Metabolic preferences [8], environmental responses [10], biofilm interactions [11••], host-cell immune interactions [12,13], and the ability to mate [14••] show significant differences between white and opaque cells. It is an attractive

\footnotetext{
(c) 2009 Elsevier Ltd. All rights reserved

*Corresponding author. Mailing Address: $60016^{\text {th }}$ Street, University of California San Francisco, San Francisco, CA 94158 Phone: 4154768783 Fax: 4155024315 ajohnson@cgl.ucsf.edu.

Publisher's Disclaimer: This is a PDF file of an unedited manuscript that has been accepted for publication. As a service to our customers we are providing this early version of the manuscript. The manuscript will undergo copyediting, typesetting, and review of the resulting proof before it is published in its final citable form. Please note that during the production process errors may be discovered which could affect the content, and all legal disclaimers that apply to the journal pertain.
} 
hypothesis that white-opaque switching evolved during C. albicans' long association with its warm-blooded host and that it plays a crucial role in the pathogen-host relationship.

\section{White-opaque switching is closely linked with mating}

White-opaque switching was discovered in 1987 by David Soll and colleagues [2••]. Its key role in the mating cycle of C. albicans was established some 15 years later [reviewed in 15, 16]. In brief, $C$. albicans' mating is controlled by transcriptional regulators encoded at the Mating Type Like (MTL) locus. There are two versions of this locus, referred to as a and $\alpha$, which contain transcriptional regulators specifying a and $\alpha$-type mating respectively. In cells containing both versions of this locus, referred to as a/ $\alpha$ cells, two homeodomain proteins (one from the a locus and one from the $\alpha$ locus) form the $\mathbf{a} 1-\alpha 2$ heterodimer which represses mating functions as well as white-opaque switching. In order to mate, an a cell must encounter an $\alpha$ cell but both cells must be in the opaque form for mating to culminate. These observations help to explain why $C$. albicans mating was so difficult to detect: more than $95 \%$ of clinical $C$. albicans isolates are $\mathbf{a} / \alpha$ strains [17] (and hence are mating and switching incompetent) and, in order to mate, $\mathbf{a}$ and $\alpha$ cells both have to undergo the rare switch to the opaque state [Figure $1]$.

\section{Multiple feedback loops contribute to stability of the two cell types}

One key characteristic of the white and opaque cell types is their stability over thousands of generations under normal laboratory conditions; that is, upon cell division, white cells almost always give rise to white cell progeny and opaque cells to opaque progeny. It has been proposed that this behavior results from the topology of the transcriptional circuit underlying the switch; this circuit consists largely of interlocking positive feedback loops [Figure 2a] [18••].

At the core of this circuit is a protein called WOR1, the first major regulator of the opaque state to be identified [19-21]. WOR1 expression is required to switch from the white to the opaque state and ectopic expression of WOR1 can drive an entire population of white cells to the opaque state. WOR 1 expression produces a direct positive feedback loop by binding its own promoter and turning on its own expression [19-21]. Activation of this feedback loop produces a forty-fold increase in WOR1 transcript levels in opaque cells compared to white cells. The WOR 1 promoter is directly repressed by the $\mathbf{a} 1-\alpha 2$ heterodimer, thus explaining the inability of $\mathbf{a} / \alpha$ cells to switch from white to opaque [Figure $2 b][9,14 \bullet \bullet, 20]$.

Three additional transcriptional regulators, EFG1, WOR2, and CZF1, complete the known

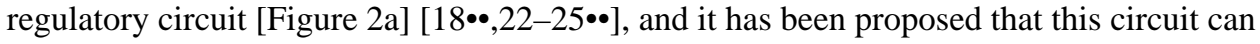
account for the major characteristics of the white-opaque switch. According to this model, the circuit is largely inactive in the white state; this is the default state [Figure 2c]. Switching occurs when the circuit becomes excited; because of the series of positive feedback loops, the circuit can remain excited for many generations [Figure 2d]. It has been hypothesized that inheritance of the opaque state results from molecules of the regulators being passed on to daughter cells following cell division; the concentrations of these regulators in the daughter cells would then be sufficiently high to re-excite the circuit and retain the opaque state. What triggers white-toopaque switching in the first place? It has been proposed that when components of the switching circuit reach a critical threshold concentration, the circuit becomes excited. In principle, this could occur through random fluctuations in the levels of a critical molecule such as WOR1. The reverse process, opaque-to-white switching, would then occur when insufficient quantities of the regulators are passed on to a daughter cell and the circuit would wind down.

Layered on this core transcriptional circuit are several chromatin modifying factors whose presence also affects switching. For example, deletion of the histone deacetylase HDA1 slightly increases switching rates from white to opaque while deletion of RPD3, another histone 
deacetylase, increases switching in both directions [26]. Recently, the acetyltransferase NAT4 and histone deacetylases HST2, SET3, and HOS2 have also been implicated in regulation of switching. Deletion of each of these genes reduced rates of white-to-opaque switching and all but HST2 increased switching from opaque to white [25••]. It is not yet clear precisely what role these chromatin remodeling factors play in the switch; one simple model is that they serve to reinforce the characteristics of the transcriptional circuit described above.

\section{Growth rate affects switching frequency}

Although the circuit described above can explain much of the behavior of the two cell types, it does not readily account for how environmental cues can affect the rate of switching. It has long been known that oxidative stress increases switching from white to opaque [10] while elevated temperatures drive switching from opaque to white [7]. Recent studies have identified several new signals that affect switching frequencies and have led to new hypotheses linking these signals to the regulatory circuit

Exposure to the DNA damaging agent methyl methane sulfonate (MMS) and deletions of the DNA repair genes RAD51 and RAD52 both increase switching from white to opaque [27•]. Hydroxyurea (HU), which delays cells in S phase of the cell cycle, also increased the whiteto-opaque switching rate. These results suggest a direct link between progression though the cell cycle and white-opaque switching. According to one model, an extended cell cycle in slow growing cells would facilitate switching by allowing WOR1 to accumulate to higher levels before being diluted by cell division; this would result in a greater proportion of cells with sufficient WOR1 to excite the regulatory circuit of Figure 2. In support of this hypothesis, depletion of the mitotic cell cycle regulator CLB4 slows cell growth and also produces increased switching from white to opaque [27•].

\section{Anaerobic growth stabilizes the opaque cell type}

Recent work has also revealed new insights into the interplay between temperature and whiteopaque switching. The observation of in vivo mating of $C$. albicans in a mouse host [28] had long presented a paradox. $C$. albicans must be opaque in order to mate [14.•] and yet opaque cells were not believed to be stable at the internal body temperature of $37^{\circ} \mathrm{C}$. However, it has recently been discovered that opaque cells are stable and capable of mating at $37^{\circ} \mathrm{C}$ if also exposed to anaerobic conditions [29]. Further work revealed that anaerobic conditions not only stabilize opaque cells but even stimulate white to opaque switching [30••]. Studies in the mouse support this view: when feces were collected from mice injected with a switching competent white strain, it was discovered that between 4 and 10 percent of cells were in the opaque form. Thus white-to-opaque switching can indeed occur at $37^{\circ} \mathrm{C}$ in a mouse model.

What is the basis for anaerobic growth stimulating white-to-opaque switching? Growth under anaerobic conditions leads to ergosterol depletion, and it has been hypothesized that this metabolic cue may signal cells to switch. In support of this view, addition of the ergosterol biosynthesis inhibitors lovastatin or ketoconazole produced an increase in switching to opaque similar to that seen under anaerobic conditions [30••].

An independent set of experiments has shown that elevated $\mathrm{CO}_{2}$ levels can also stimulate whiteto-opaque switching and can stabilize opaque cells at $37^{\circ} \mathrm{C}$ [31•]. Because $\mathrm{CO}_{2}$ levels vary dramatically among different tissues of the host, this observation strongly suggests that rates of white-opaque switching are tuned to different niches within the host. 


\section{Opaque cells may evade the innate immune system}

Differences between white and opaque cells significantly affect $C$. albicans' interactions with the host. For example, white cells appear better suited to internal infections while opaque cells thrive in skin infections [32-34]. Opaque and white cells also differ in their interactions with specific components of the host immune system. For example, opaque cells, unlike white cells, do not secrete a chemoattractant for leukocytes [12]. As a possible consequence of this difference, opaque cells are significantly less susceptible than white cells to phagocytosis by macrophage cell lines in vitro [13]. This ability to avoid the innate immune system may help C. albicans colonize internal environments where opaque cells are particularly stable. It should be stressed, however, that we know relatively little about the complete relationship between the two cell types and the host.

\section{White cells respond to pheromone by forming biofilms}

In response to the $\alpha$ mating pheromone, opaque a cells up-regulate several hundred genes, many of which are involved in preparing the cells for the subsequent steps of mating [35]. Although they appear unable to mate, white a cells also respond to the $\alpha$ pheromone $[11 \bullet \bullet$, $36]$. Both white and opaque a cells sense $\alpha$-factor through the same $\alpha$-factor receptor, STE2 [37], although white cells use a novel signaling loop not required for detection in opaque cells [38].

The response of white cells to mating pheromone is markedly different from the response observed in opaque cells. Many fewer genes are induced and, rather than preparing to mate, white cells undergo a different type of response: they form a biofilm [11••]. What could be the purpose of this response? One hypothesis is that biofilm formation facilitates subsequent mating. According to this idea, detection of pheromone allows white cells to recognize the presence of a small population of opaque cells, and by forming a biofilm, concentrates cells of opposite mating types to promote mating. Regardless of its precise role, the observation that white and opaque cells respond very differently to mating pheromone is a particularly fascinating difference between the two types of cells.

\section{Conservation of the switch components}

Although white-opaque switching appears unique to $C$. albicans and its very close relatives [39], the components that regulate the switch are conserved among many fungal species. For example, WOR1, the central regulator of white-opaque switching, is a member of a large family of fungal proteins distinguished by a highly conserved $\mathrm{N}$-terminal region [21]. Although nearly every species of fungi for which a genome sequence is available has a WOR1 homolog, the structure and biochemical function of this conserved region is not known. In all species where they have been studied, the WOR1 homologs appear to play some role in regulating morphological changes. For example, the Histoplasma capsulatum WOR1 ortholog RYP1 is a key regulator of the yeast-to-mycelial switch. RYP1 is differentially expressed in the two types of cells, binds its own promoter, and is necessary for establishment and maintenance of the yeast cell type [40•]. At this point, we can only guess how these deeply conserved transcriptional regulators became "wired-up" to catalyze white-opaque switching in $C$. albicans.

\section{Conclusion}

Despite many advances in our understanding of the regulation and role of white-opaque switching in Candida albicans, we still possess only a tentative grasp of many important aspects of this switching system. It remains to be determined whether random fluctuations in regulator levels can explain the stochastic nature of switching. The role of opaque cells in the host and 
their interaction with the innate immune system remain among the most pressing questions in this field. Specific host niches that stabilize opaque cells or promote switching from white to opaque remain largely unidentified. The exact role of biofilm formation in facilitating in vivo mating remains to be determined, as does the in vivo role of anaerobic growth and $\mathrm{CO}_{2}$. Finally, the selective pressures under which the white-opaque switch evolved remain obscure.

\section{Acknowledgments}

The authors thank Chris Cain, Sarah Foss, Aaron Hernday, and Oliver Homann for valuable comments on this manuscript. Work of the authors was supported by grants from the National Institutes of Health (AI49187) and the Ellison Foundation (ID-SS-0628-04).

\section{References}

Papers of particular interest have been highlighted as:

- of special interest

•• of outstanding interest

1. Noble SM, Johnson AD. Genetics of Candida albicans, a diploid human fungal pathogen. Annu Rev Genet 2007;41:193-211. [PubMed: 17614788]

•.2. Slutsky B, Staebell M, Anderson J, Risen L, Pfaller M, Soll DR. "White-opaque transition”: a second high-frequency switching system in Candida albicans. J Bacteriol 1987;169:189-197. [PubMed: 3539914] The discovery of white-opaque switching

3. Soll DR, Morrow B, Srikantha T. High-frequency phenotypic switching in Candida albicans. Trends Genet 1993;9:61-65. [PubMed: 8456504]

4. Johnson A. The biology of mating in Candida albicans. Nat Rev Microbiol 2003;1:106-116. [PubMed: 15035040]

5. Lockhart SR, Daniels KJ, Zhao R, Wessels D, Soll DR. Cell biology of mating in Candida albicans. Eukaryot Cell 2003;2:49-61. [PubMed: 12582122]

6. Whiteway M, Bachewich C. Morphogenesis in Candida albicans. Annu Rev Microbiol 2007;61:529553. [PubMed: 17506678]

7. Rikkerink EH, Magee BB, Magee PT. Opaque-white phenotype transition: a programmed morphological transition in Candida albicans. J Bacteriol 1988;170:895-899. [PubMed: 2828333]

8. Lan CY, Newport G, Murillo LA, Jones T, Scherer S, Davis RW, Agabian N. Metabolic specialization associated with phenotypic switching in Candida albicans. Proc Natl Acad Sci U S A 2002;99:1490714912. [PubMed: 12397174]

9. Tsong AE, Miller MG, Raisner RM, Johnson AD. Evolution of a combinatorial transcriptional circuit: a case study in yeasts. Cell 2003;115:389-399. [PubMed: 14622594]

10. Kolotila MP, Diamond RD. Effects of neutrophils and in vitro oxidants on survival and phenotypic switching of Candida albicans WO-1. Infect Immun 1990;58:1174-1179. [PubMed: 2157666]

$\bullet$ 11. Daniels KJ, Srikantha T, Lockhart SR, Pujol C, Soll DR. Opaque cells signal white cells to form biofilms in Candida albicans. EMBO J 2006;25:2240-2252. [PubMed: 16628217] Discovery that white cells form biofilms in response to mating pheromone

12. Geiger J, Wessels D, Lockhart SR, Soll DR. Release of a potent polymorphonuclear leukocyte chemoattractant is regulated by white-opaque switching in Candida albicans. Infect Immun 2004;72:667-677. [PubMed: 14742507]

13. Lohse MB, Johnson AD. Differential phagocytosis of white versus opaque Candida albicans by Drosophila and mouse phagocytes. PLoS One 2008;3:e1473. [PubMed: 18213381]

$\bullet$ 14. Miller MG, Johnson AD. White-opaque switching in Candida albicans is controlled by matingtype locus homeodomain proteins and allows efficient mating. Cell 2002;110:293-302. [PubMed: 12176317] Established the link between mating and white-opaque switching

15. Magee PT, Magee BB. Through a glass opaquely: the biological significance of mating in Candida albicans. Curr Opin Microbiol 2004;7:661-665. [PubMed: 15556040] 
16. Bennett RJ, Johnson AD. Mating in Candida albicans and the search for a sexual cycle. Annu Rev Microbiol 2005;59:233-255. [PubMed: 15910278]

17. Lockhart SR, Pujol C, Daniels KJ, Miller MG, Johnson AD, Pfaller MA, Soll DR. In Candida albicans, white-opaque switchers are homozygous for mating type. Genetics 2002;162:737-745. [PubMed: 12399384]

••18. Zordan RE, Miller MG, Galgoczy DJ, Tuch BB, Johnson AD. Interlocking transcriptional feedback loops control white-opaque switching in Candida albicans. PLoS Biol 2007;5:e256. [PubMed: 17880264] Proposed the current model for the transcriptional circuit regulating switching

19. Huang G, Wang H, Chou S, Nie X, Chen J, Liu H. Bistable expression of WOR1, a master regulator of white-opaque switching in Candida albicans. Proc Natl Acad Sci U S A 2006;103:12813-12818. [PubMed: 16905649]

20. Srikantha T, Borneman AR, Daniels KJ, Pujol C, Wu W, Seringhaus MR, Gerstein M, Yi S, Snyder M, Soll DR. TOS9 regulates white-opaque switching in Candida albicans. Eukaryot Cell 2006;5:1674-1687. [PubMed: 16950924]

21. Zordan RE, Galgoczy DJ, Johnson AD. Epigenetic properties of white-opaque switching in Candida albicans are based on a self-sustaining transcriptional feedback loop. Proc Natl Acad Sci U S A 2006;103:12807-12812. [PubMed: 16899543]

22. Vinces MD, Kumamoto CA. The morphogenetic regulator Czf1p is a DNA-binding protein that regulates white opaque switching in Candida albicans. Microbiology 2007;153:2877-2884. [PubMed: 17768232]

23. Srikantha T, Tsai LK, Daniels K, Soll DR. EFG1 null mutants of Candida albicans switch but cannot express the complete phenotype of white-phase budding cells. J Bacteriol 2000;182:1580-1591. [PubMed: 10692363]

24. Sonneborn A, Tebarth B, Ernst JF. Control of white-opaque phenotypic switching in Candida albicans by the Efg1p morphogenetic regulator. Infect Immun 1999;67:4655-4660. [PubMed: 10456912]

$\bullet$ 25. Hnisz D, Schwarzmüller T, Kuchler K. Transcriptional loops meet chromatin: a dual-layer network controls white-opaque switching in Candida albicans. Mol Microbiol. 2009 Epub. A systematic study of the impact of histone modification and chromatin remodeling on white-opaque switching frequencies

26. Srikantha T, Tsai L, Daniels K, Klar AJ, Soll DR. The histone deacetylase genes HDA1 and RPD3 play distinct roles in regulation of high-frequency phenotypic switching in Candida albicans. $\mathrm{J}$ Bacteriol 2001;183:4614-4625. [PubMed: 11443097]

-27. Alby K, Bennett RJ. Stress-induced phenotypic switching in Candida albicans. Mol Biol Cell 2009;20:3178-3191. [PubMed: 19458191]

28. Hull CM, Raisner RM, Johnson AD. Evidence for mating of the "asexual" yeast Candida albicans in a mammalian host. Science 2000;289:307-310. [PubMed: 10894780]

29. Dumitru R, Navarathna DH, Semighini CP, Elowsky CG, Dumitru RV, Dignard D, Whiteway M, Atkin AL, Nickerson KW. In vivo and in vitro anaerobic mating in Candida albicans. Eukaryot Cell 2007;6:465-472. [PubMed: 17259544]

••30. Ramírez-Zavala B, Reuss O, Park YN, Ohlsen K, Morschhäuser J. Environmental induction of white-opaque switching in Candida albicans. PLoS Pathog 2008;4:e1000089. [PubMed: 18551173] Demonstrated the impact of anaerobic growth on opaque cell stability; demonstrated white-to-opaque switching in the mouse

-31. Huang G, Srikantha T, Sahni N, Yi S, Soll DR. CO(2) regulates white-to-opaque switching in Candida albicans. Curr Biol 2009;19:330-334. [PubMed: 19200725] Discovery of a link between $\mathrm{CO}_{2}$ and white-to-opaque switching

32. Kvaal CA, Srikantha T, Soll DR. Misexpression of the white-phase-specific gene WH11 in the opaque phase of Candida albicans affects switching and virulence. Infect Immun 1997;65:4468-4475. [PubMed: 9353021]

33. Kvaal C, Lachke SA, Srikantha T, Daniels K, McCoy J, Soll DR. Misexpression of the opaque-phasespecific gene PEP1 (SAP1) in the white phase of Candida albicans confers increased virulence in a mouse model of cutaneous infection. Infect Immun 1999;67:6652-6662. [PubMed: 10569787] 
34. Lachke SA, Lockhart SR, Daniels KJ, Soll DR. Skin facilitates Candida albicans mating. Infect Immun 2003;71:4970-4976. [PubMed: 12933839]

35. Zhao R, Daniels KJ, Lockhart SR, Yeater KM, Hoyer LL, Soll DR. Unique aspects of gene expression during Candida albicans mating and possible G(1) dependency. Eukaryot Cell 2005;4:1175-1190. [PubMed: 16002644]

36. Lockhart SR, Zhao R, Daniels KJ, Soll DR. Alpha-pheromone-induced "shmooing” and gene regulation require white-opaque switching during Candida albicans mating. Eukaryot Cell 2003;2:847-855. [PubMed: 14555467]

37. Yi S, Sahni N, Daniels KJ, Pujol C, Srikantha T, Soll DR. The same receptor, G protein, and mitogenactivated protein kinase pathway activate different downstream regulators in the alternative white and opaque pheromone responses of Candida albicans. Mol Biol Cell 2008;19:957-970. [PubMed: 18162580]

38. Yi S, Sahni N, Pujol C, Daniels KJ, Srikantha T, Ma N, Soll DR. A Candida albicans-specific region of the alpha-pheromone receptor plays a selective role in the white cell pheromone response. Mol Microbiol 2009;71:925-947. [PubMed: 19170873]

39. Pujol C, Daniels KJ, Lockhart SR, Srikantha T, Radke JB, Geiger J, Soll DR. The closely related species Candida albicans and Candida dubliniensis can mate. Eukaryot Cell 2004;3:1015-1027. [PubMed: 15302834]

-40. Nguyen VQ, Sil A. Temperature-induced switch to the pathogenic yeast form of Histoplasma capsulatum requires Ryp1, a conserved transcriptional regulator. Proc Natl Acad Sci U S A 2008;105:4880-4885. [PubMed: 18339808] Identification of RYP1, a WOR1 homolog, in Histoplasma capsulatum 


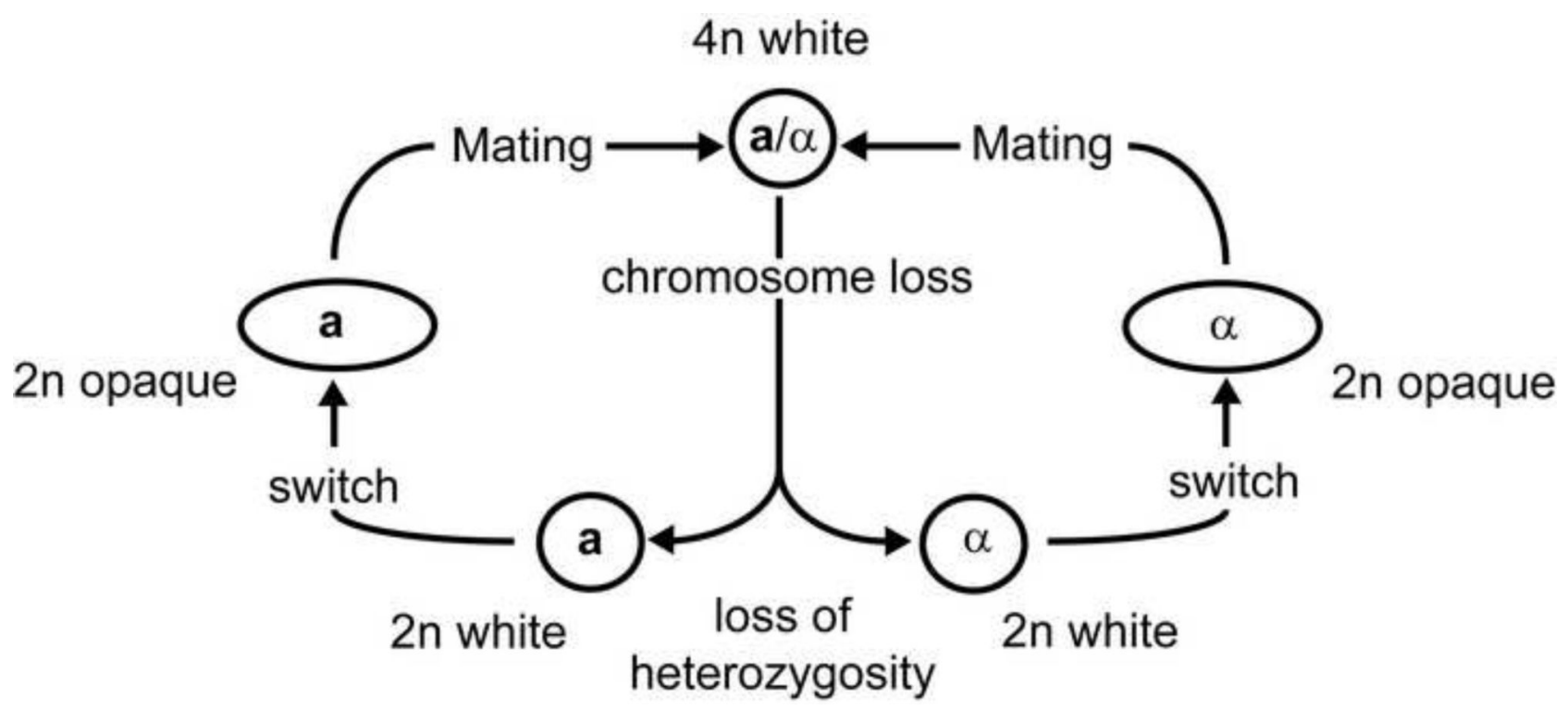

Figure 1.

Outline of the parasexual cycle of $C$. albicans. a cells mate with $\alpha$ cells, but both types of cells must be in the opaque form for this to happen. $C$. albicans is diploid so mating produces a tetraploid $\mathbf{a} / \alpha$ cell. This cell can lose chromosomes to return to the diploid state; a conventional meiosis has not been observed in $C$. albicans. 
A

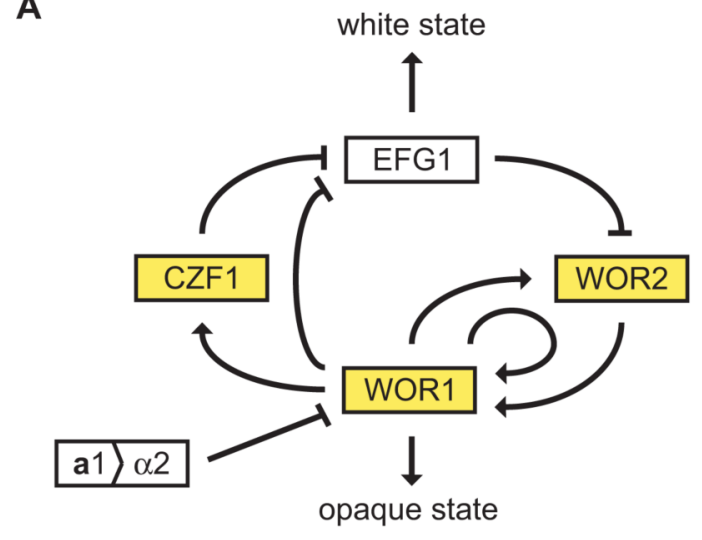

C

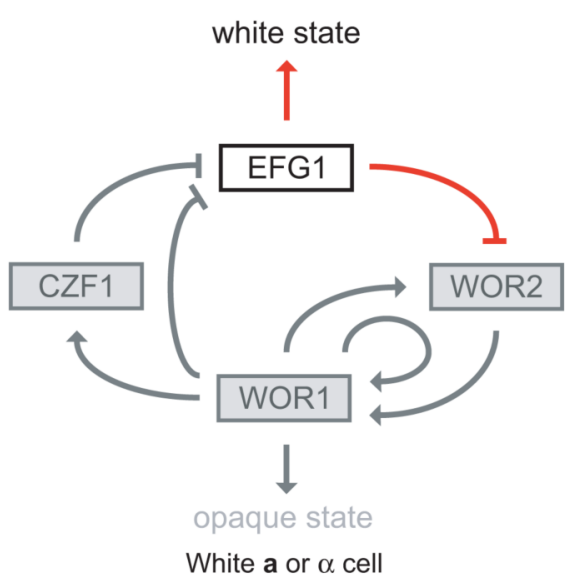

B

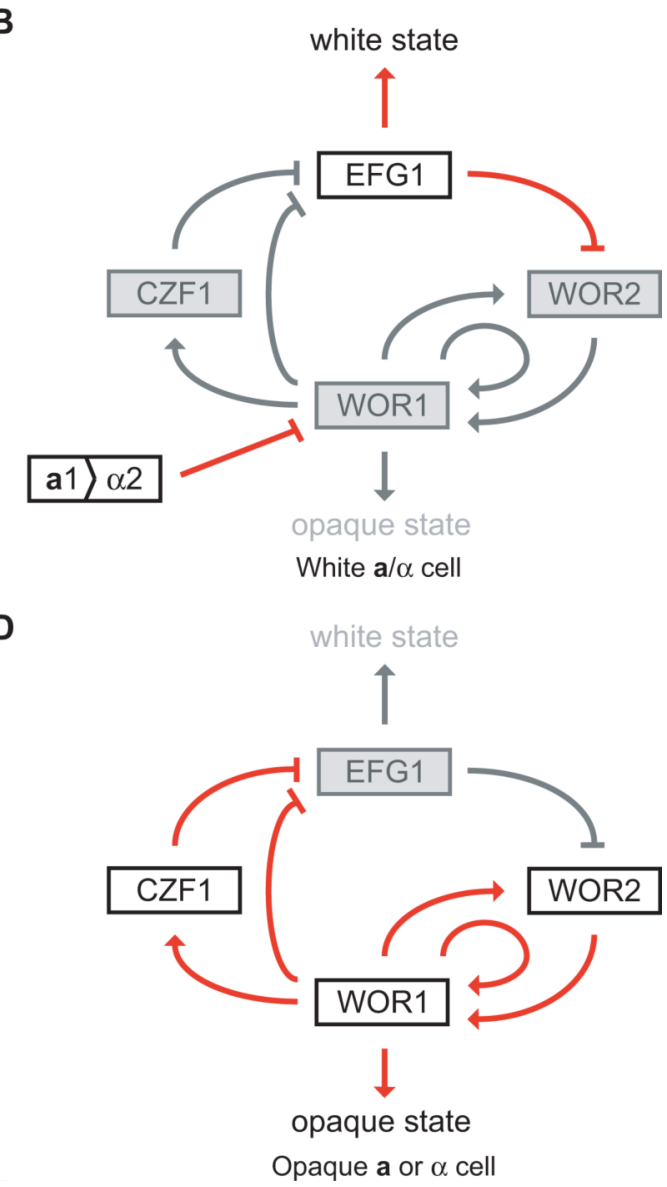

Figure 2.

Model of the white-opaque regulatory circuit and its activity in different cell types. (a) White boxes represent factors that are enriched in white cells compared with opaque cells, and yellow boxes represent opaque-enriched factors. Lines with arrows represent positive control and lines with bars, negative control. (b) White $\mathbf{a} / \alpha$ cell, with the $\mathbf{a} 1 / \alpha 2$ heterodimer repressing WOR1. This cell is locked in the white form. (c) White a or $\alpha$ cell. (d) Opaque a or $\alpha$ cell. WOR1, WOR2, and CZF1 are up-regulated relative to white cells, and EFG1 is down regulated. In panels b-d, down-regulated genes and inactive interactions are shown in gray while upregulated genes are shown in black and active interactions are shown in red. Adapted from Zordan et al 2007 [18••]. 